\title{
Eigenvalues of the Transferences of Gaussian Optical Systems
}

\section{WF Harris*}

Optometric Science Research Group, Department of Optometry, and Department of Mathematics and Statistics, University of Johannesburg, PO Box 524, Auckland Park, 2006 South Africa

\begin{abstract}
The problem of how to define an average eye leads to the question of what eigenvalues are possible for ray transferences. This paper examines the set of possible eigenvalues in the simplest possible case, that of optical systems consisting of elements that are stigmatic and centred on a common axis.
\end{abstract}

Keywords: Transference, Gaussian systems, eigenvalues, spherical systems, stigmatic systems, symplecticity

Wherever matrices find application the eigenstructure of the matrices usually turns out to be important. The eigenstructure is sometimes more closely related to the intuitive or traditional understanding of phenomena that for purposes of quantitative representation and analysis are better represented as the matrices themselves. Perhaps the best optometric example is provided by the dioptric power matrix: at least for thin systems its eigenvectors define the principal meridians and its eigenvalues are what are well known as the principal powers. The matrix is directly involved in calculations while its eigenstructure is closer to how optometry has traditionally treated dioptric power.

The ray transference has become fairly prominent recently in the optometric literature. And yet I can recall no mention of its eigenvalues and eigenvectors. My own expectation has been that it was only a matter of time before they would raise their pretty heads. So it is with no real surprise, and perhaps some satisfaction, to find that they arise in the context of the problem of calculating an average eye ${ }^{1}$. Because of the significance of the eigenvalues of ray transferences in this context it seems appropriate to take a closer look at them. Accordingly the purpose of this paper is to examine the eigenvalues of transferences for their own sake. We shall treat only the simplest case, optical systems each of whose refracting elements is both stigmatic (that is, not astigmatic) and centred on a common longitudinal axis. We call them centred Gaussian systems. The analysis gives a clearer picture of issues involved in the calculation of the average eye and suggests ways of overcoming some difficulties.

A recent paper $^{2}$ proposes a definition for an average of a set of eyes. It turns out that the definition can be justified mathematically provided none of the eyes in the set has a transference with eigenvalues that are negative real numbers. ${ }^{1}$ But what is the significance of a transference with a negative eigenvalue? What eigenvalues are possible for a transference of an optical system in general and of an eye in particular? As we shall see all eigenvalues that are possible for centred Gaussian systems are those represented in the complex plane in Figure 1. (The question of how to define an average eye, or any optical system for that matter, is not an easy one. For more on the topic the reader is referred elsewhere. ${ }^{1-6}$ )

*BSc(Eng) PhD BOptom HonsBSc(Statistics) FAAO FRSSAf

Received 29 July 2005; revised version accepted 5 December 2005 


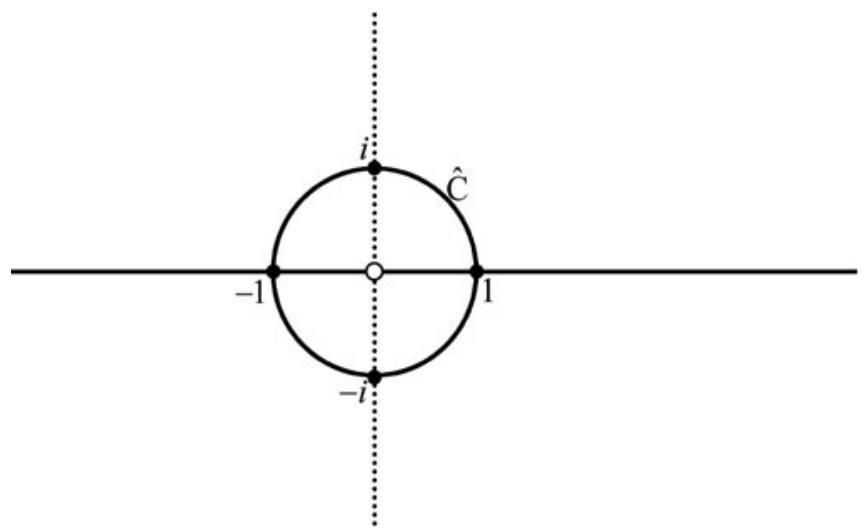

Figure 1 All possible eigenvalues of transferences in the complex plane. The eigenvalues lie on the real line excluding 0 and on the unit circle $\mathrm{C}$. The horizontal line represents the real numbers from the negative numbers on the left to the positive numbers on the right. 0 is at the origin and -1 and 1 are marked. The vertical dotted line represents the imaginary numbers; $i$, that is $\sqrt{-1}$, and $-i$ are marked. Points not on the real or imaginary number lines represent complex numbers

The transference of a centred Gaussian optical system is a $2 \times 2$ real matrix

$\mathbf{T}=\left(\begin{array}{ll}A & B \\ C & D\end{array}\right)$

with unit determinant. We define its semi-trace

$s=(A+D) / 2$.

Let $\lambda$ be an eigenvalue of $\mathbf{T}$. Then the eigenvalues of $\mathbf{T}$ are the solutions to the characteristic equation of $\mathbf{T}$, namely

$\operatorname{det}(\mathbf{T}-\lambda \mathbf{I})=0$

We write the solutions as

$\lambda_{ \pm}=s \pm \sqrt{s^{2}-1}$.

The two eigenvalues are reciprocally related,

$\lambda \_\lambda_{+}=1$,

and the semi-trace is their semi-sum,

$s=\left(\lambda_{-}+\lambda_{+}\right) / 2$

Equations 4 and 5 can both be confirmed by substitution from equation 3 .

It follows from its definition (equation 2) that $s$ can be any real number. In other words, in the complex plane, the real axis represents the set of all possible semi-traces. We use the results above to construct the set of all possible eigenvalues in the complex plane.

From equation 3 we see that the eigenvalues are real (that is, on the real axis, as shown in Figure 1) for $s^{2} \geq 1$ and not real otherwise. $\lambda_{-}$and $\lambda_{+}$are distinct for $s^{2} \neq 1$. For $s= \pm 1 \quad \lambda_{-}=\lambda_{+}= \pm 1$. For $s^{2}<1$ we rewrite equation 3 as

$\lambda_{ \pm}=s \pm i \sqrt{1-s^{2}}$

where $i=\sqrt{-1}$. From equation 3 one finds that the eigenvalues in this case have magnitude $\left|\lambda_{ \pm}\right|=\sqrt{s^{2}+\left(1-s^{2}\right)}$, that is $\left|\lambda_{ \pm}\right|=1$. In other words, for $s^{2}<1$ the eigenvalues lie on the circle $\widehat{C}$ in the complex plane with unit radius and with centre at 0 (Figure 1).

Figure 1 shows all possibilities. The eigenvalues of transferences of all possible centred Gaussian optical systems lie on the real axis, excluding 0 , and on the unit circle $\hat{C}$ centred on 0 .

The semi-trace $s$ can be plotted on the real axis. This is shown for $s=-1.8$ approximately in Figure 2. For any given $s$ the eigenvalues can be obtained by construction in the complex plane. For $s^{2}>1$ the construction is as illustrated in Figure 2. The semi-trace $s$ defines point $\mathrm{S}$ on the real line. One constructs a straight line segment ST tangent to $\hat{\mathrm{C}}$ with $\mathrm{T}$ on $\hat{\mathrm{C}}$. The rightangled triangle STO (O is at 0$)$ shows that $\mathrm{ST}$ has length $\sqrt{s^{2}-1}$. A second circle is now drawn with $\mathrm{S}$ as centre and $\mathrm{ST}$ as radius. Equation 3 then shows that $\lambda_{-}$and $\lambda_{+}$lie at the intersections of this circle and the real axis. For $s=-1$ $\lambda_{-}$and $\lambda_{+}$coincide with $s$. The same holds for $s=1$. For $s^{2}<1$ the real parts of the eigenvalues equal $s$. Thus the eigenvalues lie at the intersec-

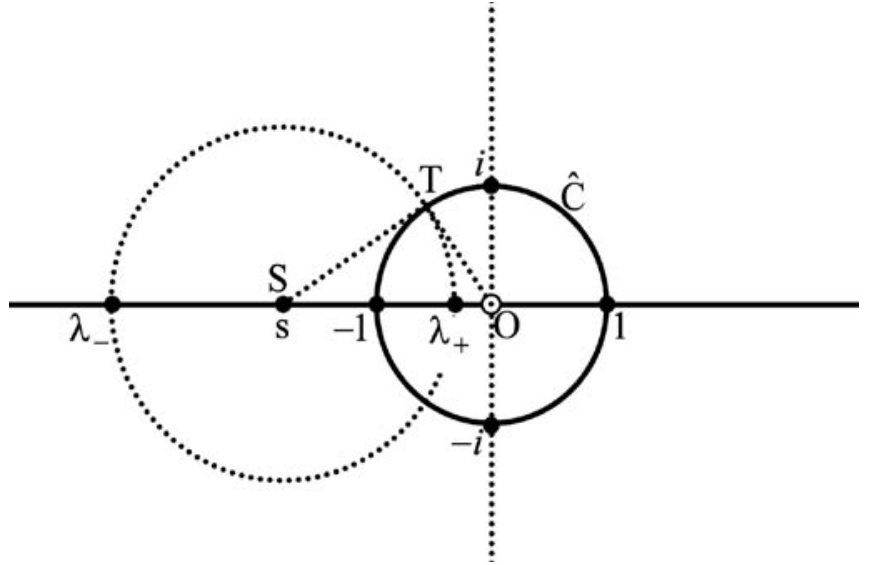

Figure 2 Constructing the eigenvalues for a particular semitrace $s<-1$. ST is tangent to the unit circle $\mathrm{C}$ at T. $\lambda_{-}$and $\lambda_{+}$ are at the intersection of the real line and the circle with centre $\mathrm{S}$ and radius $\mathrm{ST}$. 

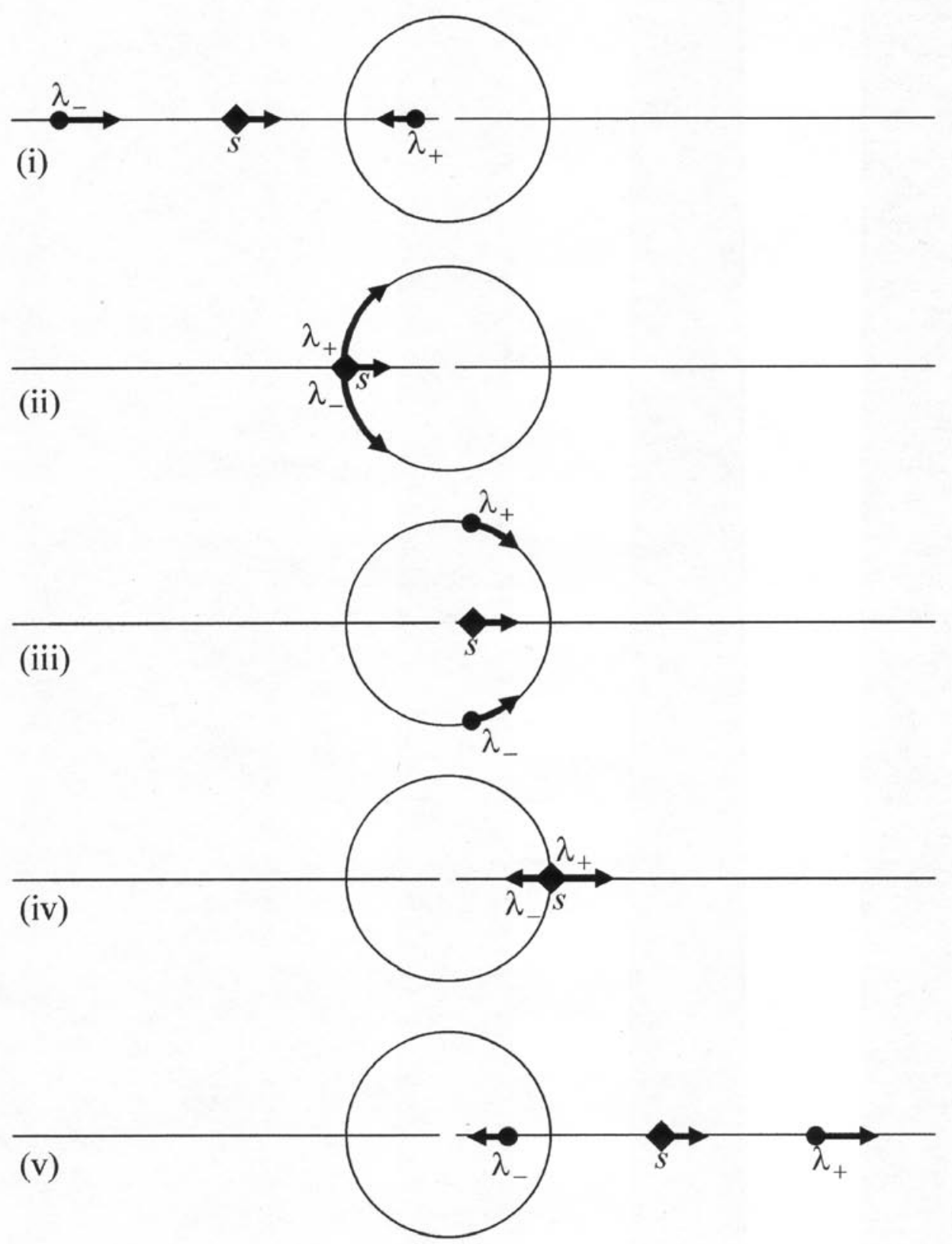

Figure 3 Behaviour of the eigenvalues $\lambda_{-}$and $\lambda_{+}$as the semi-trace $s$ increases across the real numbers. (i) is typical of Keplerian telescopes, (iii) of human eyes and (v) of Galilean telescopes. (iv) represents all thin and all elementary systems.

tion of the vertical line at $s$ and the circle $\hat{C}$ (Figure 3(iii)). In particular for $s=0 \lambda_{+}= \pm i$.

Figure 3 shows the behaviour of the eigenvalues as the semi-trace $s$ traverses its range from large negative numbers through to large positive numbers. In Figure 3(i) $\lambda_{-}$increases from $-\infty$ while $\lambda_{+}$decreases from 0 - (that is, a negative number arbitrarily close to 0$)$. $\lambda_{-}, s$ and $\lambda_{+}$meet at -1 in (ii) and then part company with $\lambda_{-}$and $\lambda_{+}$becoming complex and changing onto the bottom and top halves of the unit circle $\mathrm{C}$ respectively. The three meet again at 1 in (iv) before separating along the real axis in (v) with $\lambda$ _decreasing to $0+$ and $\lambda_{+}$increasing without limit.
All elementary systems have $A=D=1$. They are represented by Figure 3(iv).

For emmetropic eyes $A$ is 0 and for other eyes $A$ is usually close to 0 . Typically for eyes $D$ is close to 1 . Thus for eyes $s$ is around 0.5 , roughly the situation in Figure 3(iii).

For afocal systems $(C=0)$, because the transference has a unit determinant, $A D=1$. Thus the semi-trace is $s=(1 / D+D) / 2 . D$ is the usual magnification and is positive for Galilean (Figure 3(v)) and negative for Keplerian (Figure 3(i)) telescopes.

The concept of the average defined elswhere $^{2}$ is satisfactory provided none of the 
optical systems in the set has eigenvalues that are negative real numbers. We see immediately that the average will be satisfactory for a set of Gaussian telescopes, for example. We anticipate problems with Keplerian telescopes, however. Thus for a pair of telescopes (Example 1) with

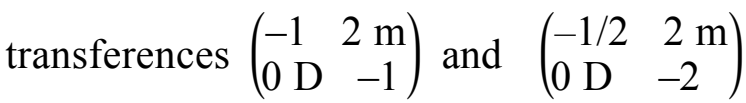

(the magnifications have magnitudes 1 and 2 respectively) the exponential-mean-log transference turns out (using MATLAB) to be the absurd value $\left(\begin{array}{ccc}0 & -4.9296 i \times 10^{8} \mathrm{~m} \\ 0 & \mathrm{D} & 0\end{array}\right)$. On the other hand (Example 2) the exponential-mean-log transference of

$\left(\begin{array}{cc}-1 / 3 & 2 \mathrm{~m} \\ 0 \mathrm{D} & -3\end{array}\right)$ and $\left(\begin{array}{cc}-1 / 2 & 2 \mathrm{~m} \\ 0 \mathrm{D} & -2\end{array}\right)$

(Keplerian telescopes with magnifications of magnitude 3 and 2 respecively) turns out to be perfectly reasonable, namely

$\left(\begin{array}{ll}-0.4082 & 1.9916 \mathrm{~m} \\ 0 \mathrm{D} & -2.4495\end{array}\right)$

(a Keplerian telescope with magnification of magnitude 2.4495).

A possible solution suggests itself in the case of Keplerian telescopes. There seems to be no good reason why one should not define a mean via the negative of transferences. In other words one could define

$\widetilde{\mathbf{T}}_{\text {neg }}=-\exp \left(\frac{1}{N} \sum_{n=1}^{N} \log \left(-\mathbf{T}_{n}\right)\right)$.

With this average one obtains the very reasonable

$\left(\begin{array}{cc}-0.7071 & 1.9629 \mathrm{~m} \\ 0 \mathrm{D} & -1.4142\end{array}\right)$ for Example 1 above,

that is, a Keplerian telescope with magnification of magnitude 1.4142. For Example 2 the answer is the same as obtained above.

Eyes have eigenvalues, like those represented in Figure 3(iii), which are some distance from the negative real axis. One expects, therefore, that the exponentialmean-log transference, as defined original-
$1 y^{2}$, will always be well defined in practice and that it will always meaningfully characterise an average eye.

\section{Acknowledgement}

This paper is based on research supported under Grant Number 2053699 to WF Harris from the National Research Foundation.

JR Cardoso of the Instituto Superior de Engenharia de Coimbra, Portugal, kindly drew my attention to the work of AJ Dragt of the Department of Physics at the University of Maryland who, in turn, kindly supplied me with his important manuscript $\mathrm{t}^{7}$.

Apart from the abstract and references to Dragt's manuscript this paper was completed on July 262005 and submitted for publication on July 29 2005. I received Dragt's manuscript $^{7}$ on July 28 2005. The core results here (essentially that represented by Figure 1) appear in Dragt's manuscript ${ }^{7}$. Dragt's application (his section 3.4) is in nuclear accelerators and makes no mention of eyes and optical systems. He makes use of the trace instead of the semi-trace.

I am particularly grateful to Drs Cardoso and Dragt. I also express my appreciation to Dr RD van Gool of the Optometric Science Research Group for reading the manuscript and for continuing discussions.

\section{References}

1. Harris WF, Cardoso JR. The exponentialmean-log-transference as a possible representation of the optical character of an average eye. Ophthal Physiol Opt 200626 in press.

2. Harris WF. The average eye. Ophthal Physiol Opt 200424 580-585.

3. van Gool RD, Harris WF. The concept of the average eye. Optom Vis Sci 200481 (12S) 163.

4. van Gool RD, Harris WF. The concept of the average eye. S Afr Optom 200564 34-43.

5. Blendowske R. The average eye. Ophthal Physiol Opt 200525459 - 460.

6. Harris WF. Author's reply. Ophthal Physiol Opt 200525 461-462.

7. Dragt, AJ. Lie Methods for Nonlinear Dynamics with Applications to Accelerator Physics. Unpublished manuscript, 2005. 